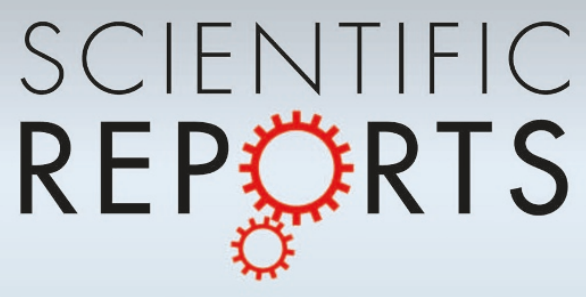

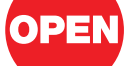

SUBJECT AREAS:

STATISTICAL PHYSICS, THERMODYNAMICS AND NONLINEAR DYNAMICS

THEORETICAL PHYSICS

MODELLING AND THEORY APPLIED PHYSICS

Received

6 March 2012

Accepted

21 June 2012

Published

02 August 2012

Correspondence and requests for materials should be addressed to G.C. Iguido. caldarelli@imtlucca.it)

\section{DebtRank: Too Central to Fail? Financial Networks, the FED and Systemic Risk}

\author{
Stefano Battiston' ', Michelangelo Puliga', Rahul Kaushik', Paolo Tasca' \& Guido Caldarellii, ${ }^{2,3,4}$
}

${ }^{1}$ Chair of Systems Design, ETH Zurich, Weinbergstrasse 56/58, 8092, Zurich, Switzerland, ${ }^{2}$ IMT Piazza San Ponziano 6, Lucca, Italy, ${ }^{3}$ Institute of Complex Systems CNR UdR Sapienza, Dip. Fisica, Piazzale Moro 200185 , Rome, ${ }^{4}$ LIMS 35a South St, Mayfair London WIK 2XF, UK.

Systemic risk, here meant as the risk of default of a large portion of the financial system, depends on the network of financial exposures among institutions. However, there is no widely accepted methodology to determine the systemically important nodes in a network. To fill this gap, we introduce, DebtRank, a novel measure of systemic impact inspired by feedback-centrality. As an application, we analyse a new and unique dataset on the USD 1.2 trillion FED emergency loans program to global financial institutions during 20082010. We find that a group of 22 institutions, which received most of the funds, form a strongly connected graph where each of the nodes becomes systemically important at the peak of the crisis. Moreover, a systemic default could have been triggered even by small dispersed shocks. The results suggest that the debate on too-big-to-fail institutions should include the even more serious issue of too-central-to-fail.

r he characterization of the architecture of economic and financial networks is gaining increasing importance ${ }^{1,2}$. Indeed, the recent economic turmoil has raised a broad awareness that the financial system should be regarded as a complex network whose nodes are financial institutions and links are financial dependencies ${ }^{3}$. In this perspective, systemic risk is meant here as the risk of a systemic default, i.e. the default of a large portion of the financial system. It can be quantified and measured from the analysis of the dynamical evolution of the nodes and from the structure of the network ${ }^{4-7}$. The main open question regarding financial networks concerns the determination of the so-called "systemically important" financial institutions, namely, the ones that, if defaulting, can trigger a systemic default and are thus to be considered "too-big-to-fail". From a network science perspective, this question is related to the concept of recursive centrality measures such as eigenvector centrality and PageRank. It is also related to the more general issue of the controllability of a complex network ${ }^{8}$. However, the investigation of how financial networks function and how systemic risk emerges is only at the beginning. The scarcity of data, due to confidentiality constraints, has limited so far the study to few national datasets ${ }^{9-12}$. The goal of this paper is to show how network science can contribute to a quantitative assessment of systemic risk. To this end, we analyse a unique and very relevant dataset by means of a novel indicator of systemic importance.

In the US, the financial crisis reached a peak in the period March 2008 - March 2010. During this time, many US and international financial institutions received aid from the US Federal Reserve Bank (FED) through emergency loans programs, including the so-called "FED Discount Window". The amount and the recipients of these loans were not disclosed until very recently (see more details in Supplementary Information, SI, Section 1-2). This data represents, to our knowledge, the first data set, publicly available, on the daily financial exposures between a central bank and a large set of institutions over several months. The data was previously analysed mainly from the point of view of accounting practice and conflicts of interests ${ }^{13}$. Here, we instead present an analysis from the perspective of complex financial networks and systemic risk.

The contributions of this paper are the following. We first analyse the portfolio of loans granted by the FED over time, both in terms of concentration and fragility. We then investigate the distribution of outstanding debt across institutions and across time. We also combine the FED dataset with data on equity investment relations among these institutions and we analyse the structure of the network of dependencies among the institutions that received funding. Finally, in order to estimate the systemic importance of the various institutions, we introduce Debt Rank. This is a novel measure, akin to feedback centrality, that takes into account in a recursive way the impact of the distress of one or more institutions to their counterparties across the whole network.

\section{Results}

Credit concentration and fragility. We start our analysis of the dataset (see Methods) from the sum of the outstanding debt across institutions, which represents the total exposure of the FED, i.e. its total potential loss in case of default of the borrowers. This number rose very sharply in November of 2008, up to around USD 1.2 

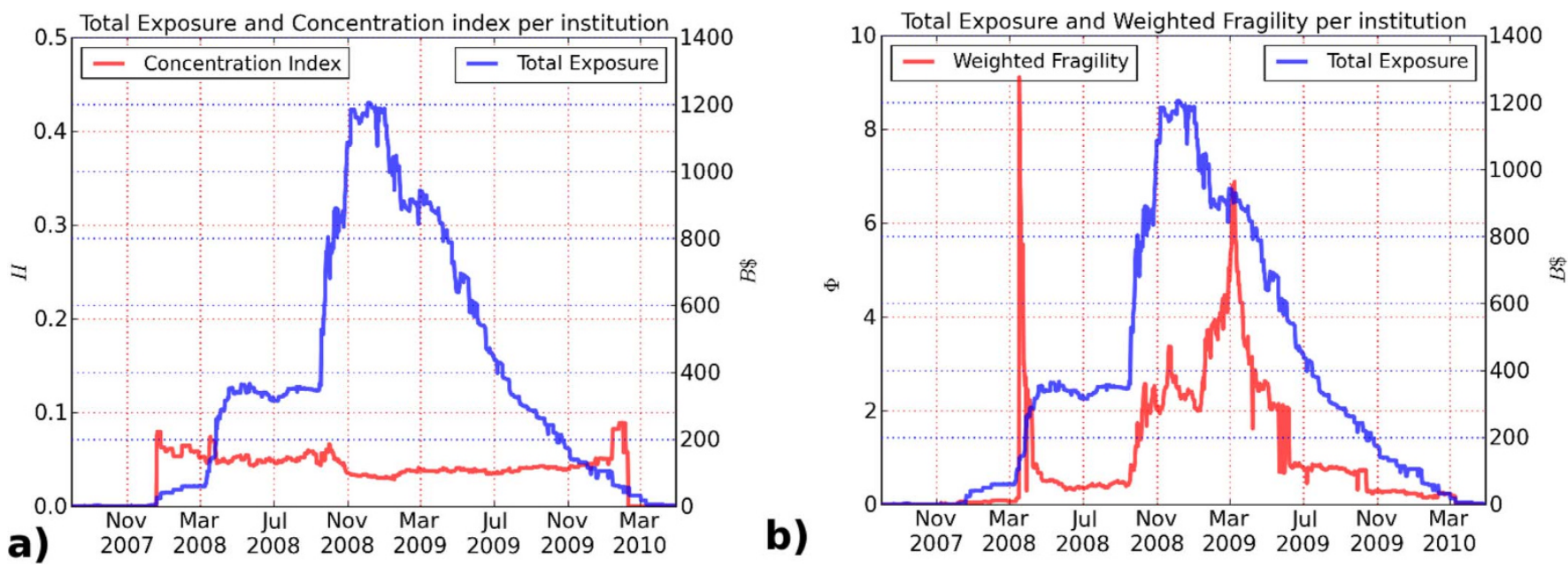
Total Exposure and Weighted Fragility per institution

Figure 1 Exposure, concentration and fragility of the FED. (a) Plot over time of the total exposure of the FED (blue) and of Herfindhal index H of the FED credit portfolio (in red). The effective number of borrowers is given by the reciprocal of $\mathrm{H}$, which varies between 10 and 30 . (b) Plot over time of the total exposure of the FED (blue) and of fragility the FED credit portfolio (in red). The peaks correspond to major distress events. E.g., the rescue of Bear Sterns in March of 2008, the Citigroup big losses (USD 2.8 billions) in October of 2008 and the market drop of March, 2009. Note that in this picture we removed big peak in fragility caused by the shock of the Lehman and Brothers default (for more details see SI).

trillion, from where, in the following 12 months, it steadily decreased down to few billions (Fig. 1a). As a measure of concentration of the credit portfolio, we compute the Herfindhal index, $H$, of the outstanding debts (see Methods). As shown in Fig. $1, H$ remains around 0.05 , meaning that, despite the large variation in the size of the exposure, the number of leading borrowers remained approximately around 20 . What did not remain constant over time is the fragility of the leading borrowers, defined as debt-to-equity ratio. The weighted average, $\Phi(t)$, of the fragility, which reflects the fragility of the largest borrowers, displays peaks in correspondance to the major events, such as the Bear Sterns case (March of 2008) and the drop in stock market value as of March of 2009 (Fig. 1b). Remarkably, when the total exposure of the FED still amounted to 900 billions, the debt of the leading borrowers was more than 6 times larger than their market capitalization.

Debt distribution. The dataset provides a unique opportunity to study the distribution of the outstanding debt across institutions and across time. Based on the behaviour of the total FED exposure, we divide the time span of the data into 3 phases (see caption Fig. 2a). In principle, we could study the daily evolution of the distribution. In practice, on a single day the number of institutions with debt to the FED is not always large enough to reveal its underlying distribution. To improve the statistics, we pool the values of debt over intervals of 30 days, after checking for the homogeneity of the debt time series within those intervals (see also Sec. 2 of SI). As shown in the inset of Fig. $2 \mathrm{a}$, we choose ten representative such intervals so that each phase is covered. Fig. 2a shows the complementary cumulative distribution of outstanding debt, computed in each of the 30-day intervals. The values of debt are rescaled by the total debt across institutions. The hypothesis that the distribution of debt across institutions is a power law is rejected by statistical tests in all periods (see also SI, Sec. 2.1). This contrasts with what was found in previous studies on the distribution of $\mathrm{debt}^{9,14}$, and more in general in various economic contexts $^{15,16}$. However, here we are in presence of emergency loans for which not every firm is eligible. It is thus likely that small institutions are under-represented in this dataset and that there is also an upper limit on the loans, which would explain the observed deviation from a power law (see also SI, Sec. 2).

Statistical tests suggest that the functional form of the distributions is the same across periods and that their parameters are more similar within the same market phase (see also SI, Sec. 2). This can be seen also in Fig 2.a where the curves in blue and violet cluster together. This is a remarkable finding, in particular during the third phase, since the total amount of debt to the FED varied in time by almost one order of magnitude (from USD 1200 billion in January of 2009 to less than USD 200 billions in October of 2009). A trivial explanation of the scaling behaviour would be that at each point in time the FED lends to all institutions in the same proportion and that only the total amount varies. The analysis of the outstanding debt pattern over time across the top 100 borrowers, shown in Fig. 2b, rules out this explanation. We find that although about 30 institutions reached their respective peak of debt in the same period, many others had their peak few months earlier or later. Moreover, while for some institutions the debt declined rapidly, for others it declined slowly. Given the heterogeneity across the debt patterns (Fig. 2b), the similarity of the debt distributions seems to be an emerging property of the system. Further evidence of this property comes from complementary data on the FED balance sheet. We find that the equivalent amount that was paid back by the institutions reappears on the FED balance sheet as mortgage backed securities (see SI, Fig. 1b). This suggests that as banks started to pay back their debt, the FED began to buy from them the toxic assets which were at origin of the crisis (more details in SI, Section 3).

Network analysis. In a network perspective, nodes represent institutions, while directed edges represent lending relations weighted by the amount of the outstanding debt. The network has a simple structure (a star with the FED in the center, see also SI, Fig. 2a-11a), although it is known that these institutions had also many financial dependencies among each other. Indeed, it is part of the normal business of financial firms to invest in other firms by utilizing various lending mechanisms ${ }^{9-12}$ and by acquiring equity stakes (although these forms of investment cannot be regarded as equivalent). Unfortunately, information on the former type of investment is not available because of confidentiality issues, especially because these are global firms from different countries. However, previous work has shown that the equity investment network of transnational corporations (TNC) has a bow-tie structure with financial firms in the core forming a tightly connected structure ${ }^{3}$.

We thus match the FED dataset with data on equity investment relations at the end of 2007 (i.e. at the beginning of the FED discount 

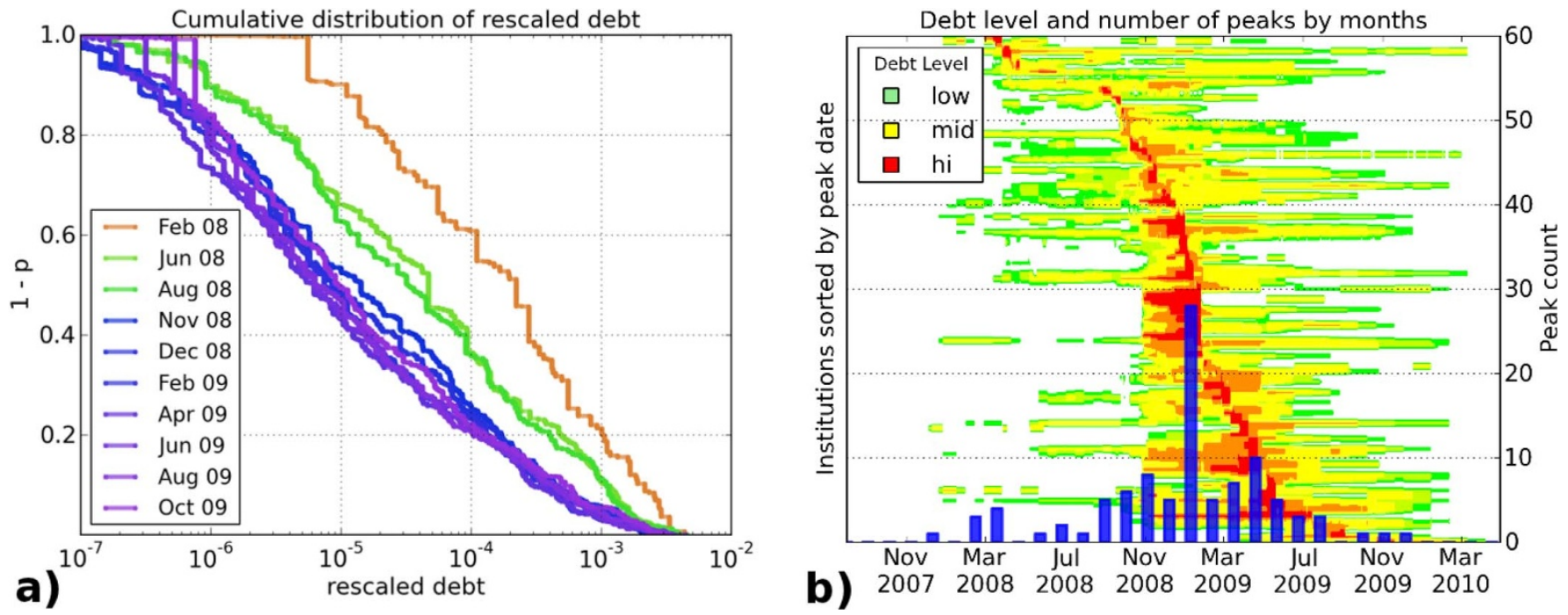

Figure $2 \mid$ Distribution and temporal patterns of debt. (a) We split data into three phases: (1) Beginning of the crisis (from August, 2007 until April, 2008), where the FED's exposure is below USD 20 billion; (2) Plateau (April, 2008 till October 2008), where the exposure raises and stays around 380 billion for 3 months; (3) Peak and decline (October, 2008 until April, 2010), where the exposure peaks in a short span of time and starts to decline at a slower rate, due to a parallel toxic asset purchasing program (see SI, Section 3) We have chosen ten representative periods of 30 days, as shown in the inset. For each period, debt values across days were pooled together in order to increase the statistics (under the assumption that such data are generated by the same stochastic process). The main plot shows the empirical complementary cumulative distributions of outstanding debt, computed in each of the 10 periods. For each distribution, the values of debt are rescaled by the total debt across institutions in that period. Note that distributions within the same phase look similar. (b) The debt of the top 100 institutions (by average debt) is represented as a topographic map where the color indicates the debt of each institution as a percentage of its own maximum debt: from green to yellow, to red (respectively, $0-30 \%, 30-70 \%$, $70-100 \%$ ). The institutions are sorted by their peak date, from upper left to bottom: institutions having an earlier peak are in the upper left corner of the plot. The inset figure shows an histogram counting how many institutions were in their peak of debt during each month. Note that in the worst case almost 30 institutions where in their maximum level of debt at the same time, thus indicating a high degree of synchronicity.

window data time span). We then focus on the 22 institutions that received, on average over the 10 periods, more than USD 5 billion. The top borrowing institutions accounted, at the peak of the crisis, for a total of USD 804 billion out of USD 1.2 trillion of funding. Interestingly, all these institutions turn out to be in the core (i.e., the largest strongly connected component) of the TNC network mentioned above. Moreover, the subnetwork of these 22 institutions is itself strongly connected (which would not need to be true). This means that each of the 22 institutions in the network can reach any other via a directed path. The overwhelming majority of pairs of nodes $(99.1 \%)$ are at distance 1 or 2 from each other. The structure is relatively homogeneous with a high density of connections. The average out-degree and in-degree are, respectively, $12.1 \pm 5.7$ and $12.1 \pm 3.2$ (the average must coincide although the network is not symmetric). The density of links is 0.58 . The k-core analysis yields a core number equal to 8 for all nodes except 3, with an average of 7.7. This finding suggests, in the lack of alternative information, to take as a proxy of the financial dependencies among institutions the network of their mutual equity investments, appropriately normalised (see Methods).

DebtRank and systemically important institutions. In order to estimate the impact of a node on the others, we then introduce DebtRank, a novel measure inspired by feedback centrality, that takes recursively into account the impact of the distress of an initial node across the whole network. DebtRank of node $i$, denoted as $R_{i}$, is a number measuring the fraction of the total economic value in the network that is potentially affected by the distress or the default of node $i$ (see Methods). DebtRank can be used to construct a ranking, but it is not itself the rank of the node. Its computation differs from the methods based on the default cascade dynamics ${ }^{11,12,17}$ in which, below the threshold no impact is propagated to the neighbors ${ }^{18}$ (see
SI, Section 3.1). Feedback centrality measures ${ }^{19}$ have found successful applications in many domains ranging from rankings in the worldwide-web (e.g. PageRank) to corporate control in economic networks ${ }^{3}$. Feedback centrality has a physical analogy with the in-flow in a non-homogeneous diffusion process (see SI, Section 3.1). In presence of a cycle in the network there is an infinite number of reverberations of the impact of a node to the others and back to itself, which leads to no simple and measurable economic interpretation. DebtRank overcomes this problem by only allowing for walks that do not visit the same edge twice.

We apply the computation of DebtRank on the network of the 22 top borrowers of the FED by carrying out two experiments. In the first one, for each node $i$, we compute DebtRank in the case of its default (i.e., $\psi_{i}=1$, see Methods). Notice that in the impact matrix used by DebtRank we take into account the level of market capitalization of the nodes (see Methods), thus obtaining a set of values of $R_{i}$ in each period. The diagrams in Fig. 3 display the structure of the network and allow, at the same time, to compare the values of DebtRank of any pair of institutions. A high value of DebtRank corresponds to a more central location of the node. Therefore, the terms "DebtRank" and "centrality" will be used interchangeably in the following. As it can be seen from the figure, at the peak of the crisis all nodes became more central, meaning that the default of each of them would cause a larger economic loss in the network. Figure 4 shows instead how the DebtRank of each institution varies with its total asset, relative to the sum of total assets in the network. The color of the bubbles reflects the fragility of the institutions (i.e. debt over equity ratio). We observe that towards the peak of the crisis most institutions have high level of fragility and in addition high values of DebtRank. This implies that each bank was more likely to default on its own, and, moreover, that in case of default, it would cause a larger loss. We find that even in a conservative scenario 

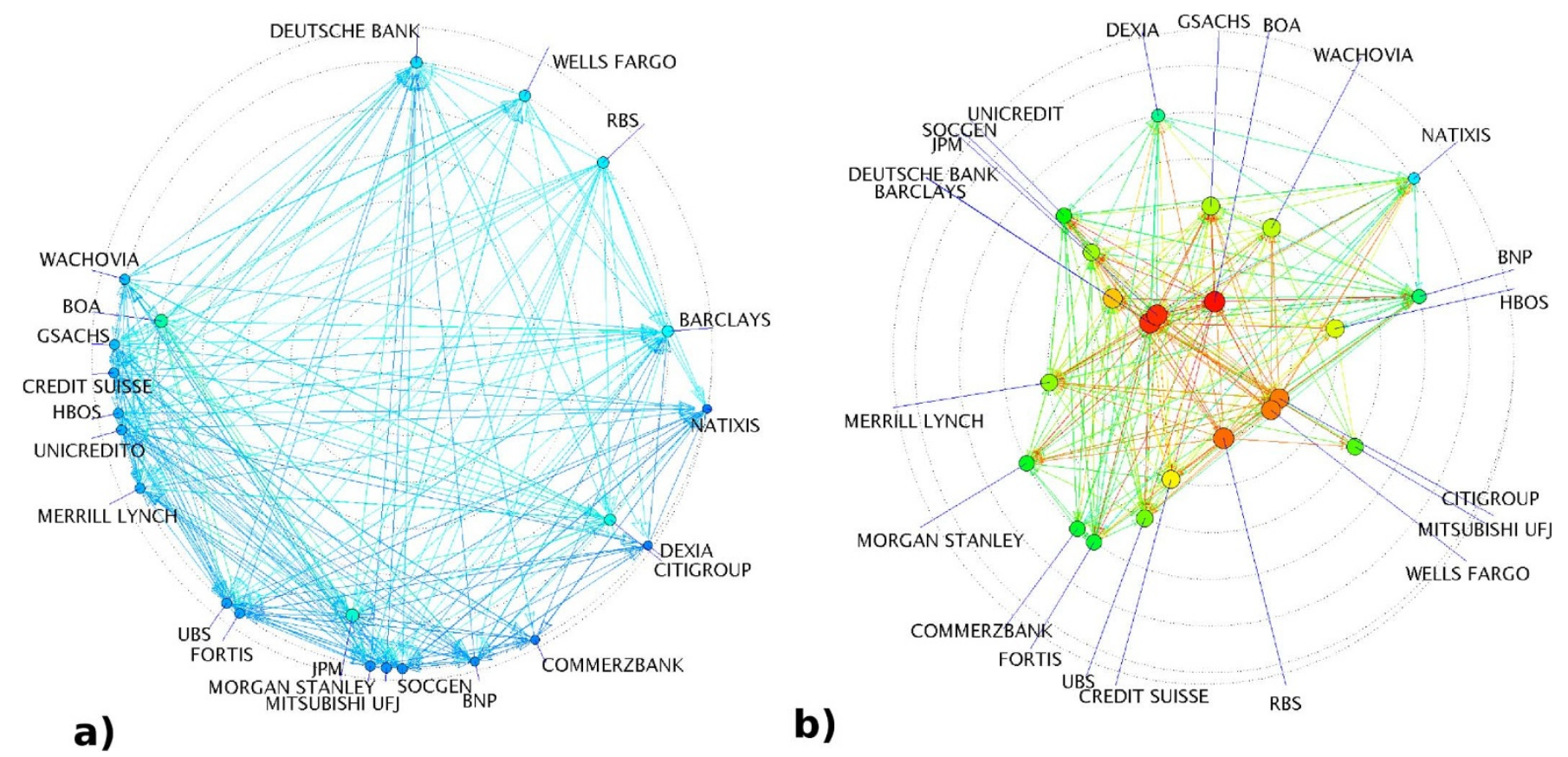

Figure $3 \mid$ The network of the top borrowers. Nodes represent financial institutions (selected as explained in the text). Outgoing links represent the estimated potential impact of an institution to another one (see Methods). The nodes are positioned within a circle of radius 1 , centred in 0 . The distance of each node from the center is $1-R^{(D)_{i}}$, while the angle increases linearly with $R^{(D)}$ from 0 to $10 \pi$. Thus, the closer a node is to the center the higher is its DebtRank (the intuition here is its centrality). A node in the center (DebtRank $=1$ ) is able to put under distress the entire economic value of the network. DebtRank decreases by moving outwards and leftwards along the spiral. The diagram allows at the same time to visualise the structure of the network and to compare the importance of any two given nodes. The size and the color of the node reflects the DebtRank value (larger and red nodes have higher DebtRank). The color of a link reflects the DebtRank of the node from which it originates (red links originate from node with high DebtRank and make high impact to the destination nodes). (a) Period one, at the beginning of the crisis (see also inset of Fig. 2a). Most of the nodes have low levels of DebtRank, i.e., they are located close to the border. (b) Period four (peak of the crisis). Nodes have comparable levels of Debt Rank. However, they are also much more central, i.e., they can impact a large fraction of the total economic value. A single default is likely to trigger a systemic failure.
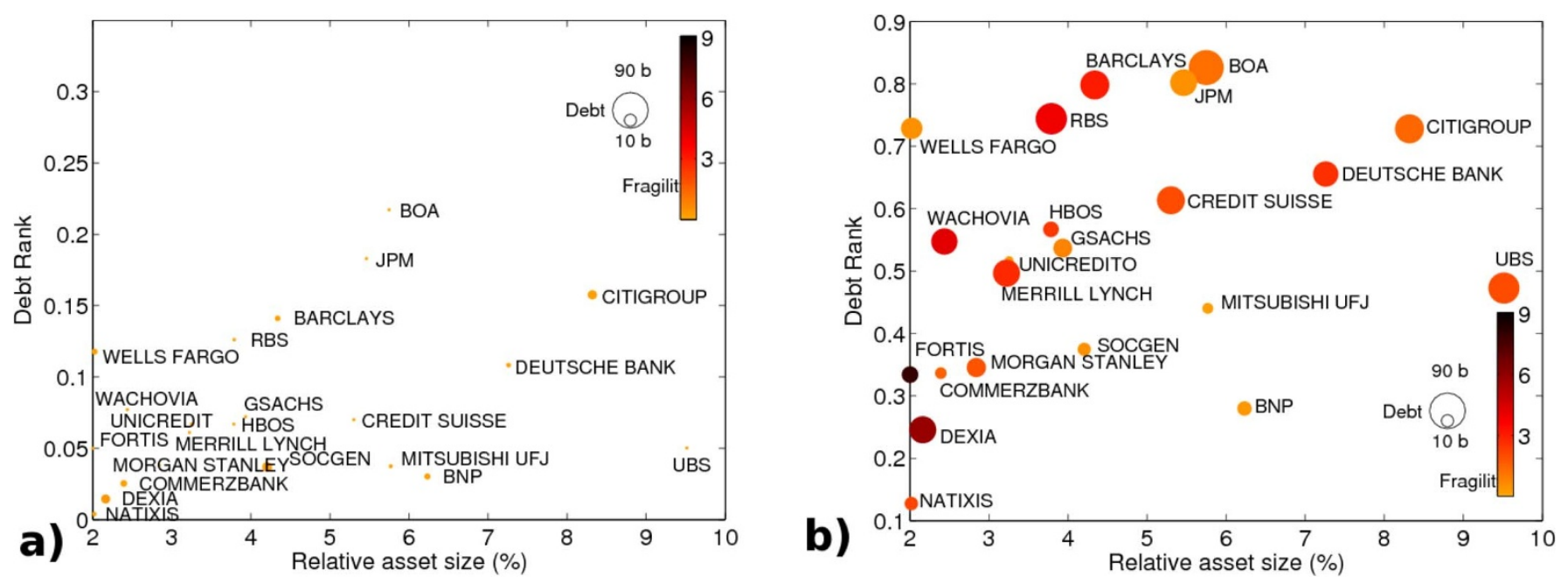

Figure $4 \mid$ Debt Rank, asset size and fragility. Scatter plot of DebtRank versus asset size, measured as a fraction (in \%) of the total of the asset size in the network. For sake of simplicity, in the experiment, asset size was assumed constant during the time span of the data. Notice that institutions such as UBS, or CITIGROUP alone account for almost $10 \%$ of the total assets. The size of each bubble is proportional to the outstanding debt of the institution while the color reflects its fragility, defined as the ratio of debt over market capitalization in the given period, as in the previous section. (a) Period one. Since the outstanding debt was very low or zero, most nodes appear small and have levels of DebtRank below 0.3, but comparable among each other. (b) Period four. Many institutions have a Debt Rank larger then 0.5 , i.e. each can impact, alone, the majority of the economic value in the network. The outstanding debt in this period is close to the peak for all the institutions, as reflected by the size of the bubbles. Notice, also a higher fragility, most bubbles are red, although with some heterogeneity. 
(e.g. we assume that in the first months of 2008 each bank had enough core capital to withstand at least the default of 5 counterparties, $\alpha=0.2$, see also Methods), most of the 22 institutions become very systemically important at the peak of the crisis (Fig. 3b). In period 1, all institutions have a DebtRank below 0.25 with an average value of $0.08 \pm 0.06$ (Fig. 4a) and are not very central (Fig. 3a). In contrast, in period 4 (average $0.52 \pm 0.20$ ), many institutions are able, alone, to affect more than the $70 \%$ of the total value in the network (Fig. 4b), despite the fact that in terms of asset size they represent less than $10 \%$ of the total.

In the second experiment, we compute DebtRank in the situation in which none of the nodes in the network defaults initially but all are at level $\psi<1$ of distress (see Methods). We refer to this case as Group DebtRank. We find that the initial shocks always generate an impact larger than the shocks themselves even in the periods preceding the peak of the crisis. For instance, with $\psi=0.1$, i.e. an initial shock of $10 \%$, the total loss varies across periods from a minimum of $22 \%$ to a maximum of $65 \%$ (see SI, Section 3.6). Notice, that the mechanism differs from the contagion found in epidemic spreading ${ }^{20}$ and other contact processes. Here, the impact of an institution $i$ to another, $j$, depends both on the relative exposure of $j$ to $i$ and on the ratio between such exposure and the capital buffer of $i$ (see Methods). It follows that the impact of $i$ over $j$ can be larger then the relative exposure of $j$ to $i$ (the impact matrix is not column stochastic). Indeed, financial institutions, by seeking for profit, tend to be highly leveraged, i.e., to have small capital compared to total assets. This is how the system can end up in a situation in which a small loss can cause a systemic default.

It is important to emphasise that there is more to DebtRank than size. The correlation between asset size and DebtRank is always lower than 0.4 and decreases towards the peak of the crisis (see SI, Section 3.7). We have also compared DebtRank with the measures of systemic importance obtained both with the default cascade dynamics and with two feedback centralities, including the classic eigenvector centrality. In a nutshell, all measures differ in the timing and in the magnitude of their response. Although the ranking provided by the four measures are similar (implying that these measures are consistent among each other), DebtRank is the only one that delivers a clear response well before the peak of the crisis (see SI, Sec. 3.5). This feature makes it a better candidate as a possible earlywarning indicator. Moreover, DebtRank has the precise meaning of economic loss, measured in dollars, caused by the distress or default of a node.

\section{Discussion}

In this paper, we presented the first complex networks analysis of the controversial FED emergency program dataset. We find that, while the number of leading borrowers to the FED only varied between 10 and 30 , the fragility of the portfolio reflected closely the major events of distress in the market. We also find that the distribution of debt across institutions has the same functional form over time. In order to estimate the systemic importance of a node, or a group of nodes, we introduce DebtRank, a novel metric that, similar to PageRank and other feedback centrality measures, takes into account recursively all the paths in the network. However, differently from these, DebtRank avoids the infinite reverberation by excluding the walks in which edges are repeated. By matching the FED data with data on equity investments relations in the same period, we find that the largest borrowers were interconnected in a dense network. Each of them was very central and could impact significantly every other node in only one or two steps. Moreover, we find that a small shock to the system as whole could get amplified by the many paths in the network into a systemic default. These findings should be regarded with caution since the network of impact used for the experiments is a proxy of the real, unknown, one. However they demonstrate what kind of insights can be gained with our methodology. The work contributes to the understanding of financial networks, but the methodology is relevant to the field of Complex Networks in general, since it can be applied to detect systemically important nodes in any directed and weighted network.

The methodology is also relevant to a broader audience of regulators and risk management practitioners since it provides a quantitative assessment of the systemically important financial institutions. In this respect, our results suggest that the current public discussion on too-big-to-fail institutions should be broadened to the networktheory notion of too-central-to-fail, so to account for the existence of densely connected cores in financial networks.

\section{Methods}

Data. Data consists of daily time series of outstanding debt and market capitalization of 407 institutions in a period of 1004 days spanning from August 2007 to June 2010. Outstanding debt refers here to the amount owed by an institution to the FED as a result of the emergency loans granted by the FED via the so-called Discount Window and various emergency programs. This means that these were loans for which the FED was lender-of-last-resort. The dataset analysed here is the one released by Bloomberg after consolidating the original dataset released by the FED. We have also obtained and analysed the original dataset (for more information, see SI Section 1). This data is unique since information on this kind of loans were never disclosed before. In addition, they refer to the crucial phase of the financial crisis. The data on equity investment were obtained from the Orbis Database of Bureau Van Dijk and were analysed in a previous study ${ }^{3}$. They include the equity shares owned by a firm in another one in the last quarter of 2007.

Credit portfolio concentration. The Herfindhal index is a common measure of size concentration. In our context, we define it as

$$
H(t)=\frac{\sum_{i=1}^{n} d_{i}(t)^{2}}{\left(\sum_{i=1}^{n} d_{i}(t)\right)^{2}},
$$

where $d_{i}(t)$ denotes the outstanding debt of borrower $i$ at time $t$. When the debt is equally distributed among $n$ institutions, $H=1 / n$. In contrast, when all the institutions except one have zero debt, then $H=1$. Thus, the inverse of $H$ is a measures of the number of leading borrowers. For instance, in the case of 3 institutions with debt values equal to $0.8,0.1,0.1$, the Herfindhal index is $H=0.8^{2}+$ $0.1^{2}+0.1^{2}=0.66$ and $H^{-1}=1.5$.

The higher the debt (relative to the market capitalization of an institution), the weaker it is the institution, financially speaking. Thus, the ratio $\phi_{i}(t)=d_{i} / e_{i}$ captures its financial fragility at time $t$. The following weighted average gives then a measure of fragility of the entire FED credit portfolio:

$$
\Phi(t)=\frac{\sum_{i=1}^{n} \phi_{i}(t) d_{i}(t)}{\sum_{i=1}^{n} d_{i}(t)},
$$

The debt-weighted average ensures that the fragility values of the institutions with larger debt count more.

DebtRank. Methodology. We introduce a directed network in which the nodes represent institutions and the links represent financial dependencies. We denote the amount invested by $i$ in the funding of $j$ as $A_{i j}$. Thus, $A$ is the weighted adjacency matrix of the investment network. The total value of the asset invested by $i$ in funding activities is $A_{i}=\Sigma_{l} A_{i l}$. We denote by $E_{i}$ the capital of $i$ (more precisely, the so-called "tier 1 capital"), which represents the capital buffer of $i$ against shocks ${ }^{11,12}$. When $E_{i} \leq \gamma$ the firm defaults, where $\gamma$ is a positive threshold. Further details on the structure of the balance sheet in relation to a financial network can be found in previous work ${ }^{17}$. If the node $i$ defaults, the node $j$ faces a loss of $A_{j i}$ (we exclude, in the short run, any recovery of the assets invested in $i$ ), and the node $j$ also defaults if $A_{j i}>E_{j}$. We will account later on for the case of the node $j$ not defaulting but going only under distress. We define the impact of $i$ on $j$ as $W_{i j}=\min \left\{1, A_{j i} / E_{j}\right\}$. Thus, if the loss exceeds capital, the impact is 1 . Notice that the matrix $W$ is, in general, neither column-stochastic nor row-stochastic. We further take into account the economic value of the impact of $i$ on $j$ by multiplying the impact by the relative economic value of the node $j, v_{j}=A_{j} / \Sigma_{l} A_{l}$ (other proxies could be taken for $v_{j}$ ). The value of the impact of $i$ on its neighbours is then $I_{i}=\Sigma_{j} W_{i j} v_{j}$. It measures the fraction of economic value in the network that is impacted by $i$ directly.

We now want to take into account the impact of $i$ on its indirect successors, that is, the nodes that can be reached from $i$ and are at distance 2 or more. In analogy to the feedback centrality we could define the impact in terms of the following recursive equation $I_{i}=\Sigma_{j} W_{i j} v_{j}+\beta \Sigma_{j} W_{i j} I_{j}$, where the second term accounts for the indirect impact via the neighbours. The parameter $\beta<1$ is a dampening factor. In vector notation, we have $I=W v+\beta W I$, which yields $I=(\llbracket-\beta W)^{-1} W v=\sum_{k=0}^{\infty}\left(\beta^{k} W^{k}\right) W v$, as long as the largest eigenvalue of $W$ is smaller than $1 / \beta$.

One result of the graph theory is that in the power $k$ of the adjacency matrix the element $i j$ amounts to the number of walks of length $k$ from $i$ to $j$. In the case of a weighted matrix, it amounts to a sum across the walks of length $k$ from $i$ to $j$ of the product of the weights along each walk ${ }^{15}$. Longer walks contribute less to the centrality because of the dampening factor $\beta^{k}$. However, if two nodes $i$ and $j$ are connected in a 
cycle $\left(W_{i j}>0\right.$ and $\left.W_{j i}>0\right)$, the impact of node $i$ to $j$ hits back on $i$ and keeps cycling an infinite number of times (although with dampening). A single reverberation of the impact of $i$ back to itself is realistic and mathematically acceptable. Further reverberations lead instead to an inconsistency because the impact could become larger than one. The reason is that if the impact walks its way several times through a cycle, then we are counting the impact of a node on another one more than once. The same problem applies also to any cycle not involving $i$, but located downstream of $i$ in the network. Removing the cycles altogether from the network and considering its corresponding acyclic graph would remove entirely the reverberation and cut many links, thus strongly underestimating the impact.

In contrast, in this paper, we propose the following approach. We keep the network as it is, but we exclude the walks in which one or more edges are repeated. A convenient way to do this (a sufficient condition) is to introduce the following process. To each node we associate two state variables. $h_{i}$ is a continuous variable with $h_{i} \in[0,1]$. Instead, $s_{i}$ is a discrete variable with 3 possible states, undistressed, distressed, inactive: $s_{i} \in\{U, D, I\}$. Denoting by $S_{f}$ the set of nodes in distress at time 1 , the initial conditions are: $h_{i}(1)=\psi \forall i \in S_{f} ; h_{i}(1)=0 \forall i \notin S_{f}$, and $s_{i}(1)=D, \forall i \in S_{f} ; s_{i}(1)=U \forall i \notin S_{f}$. The parameter $\psi$ measures the initial level of distress: $\psi \in[0,1]$, with $\psi=1$ meaning default. The dynamics is defined as follows,

$$
\begin{gathered}
h_{i}(t)=\min \left\{1, h_{i}(t-1)+\sum_{j} W_{j i} h_{j}(t-1)\right\} \text {, where } j \mid s_{j}(t-1)=D, \\
s_{i}(t)= \begin{cases}\mathrm{D} & \text { if } h_{i}(t)>0 ; s_{i}(t-1) \neq I \\
I & \text { if } s_{i}(t-1)=D \\
s_{i}(t-1) & \text { otherwise, }\end{cases}
\end{gathered}
$$

for all $i$ and for $t>=2$, where all variables $h_{i}$ are first updated in parallel, followed by un update in parallel of all variables $s_{i}$. After a finite number of steps $T$ the dynamics stops and all the nodes in the network are either in state $U$ or $I$. The intuition is that a nodes goes in distress when a predecessor just went in distress and so recursively. The fraction of propagated distress is given by the impact matrix $W_{i j}$. Because $W_{i j} \leq 1$ the longer the path from the node i initially in distress and node $j$, the smaller is the indirect impact on $j$. Notice that a node that goes in the $D$ state, will move to the $I$ state one step later. This means that if there is a cycle of length 2 the node will not be able to propagate impact to its successor more than once. This condition satisfies the requirement, mentioned earlier, of excluding the walks in which an edge is repeated. An illustration on a simple example is provided in the SI, Section 3.2.1.

The DebtRank of the set $S_{f}$ is then defined as

$$
R=\sum_{j} h_{j}(T) v_{j}-\sum_{j} h_{j}(1) v_{j},
$$

i.e., R measures the distressed induced in the system, excluding the initial distress. If $S_{f}$ is a single node the DebtRank measures the systemic impact of the node on the network. In this case, it is of interest to set $\psi=1$ and to see the impact of a defaulting node. If $S_{f}$ is a set of nodes it can be interesting to compute the impact of a small shock on the group. Indeed, while it is trivial that the default of a large group would cause the default of the whole network, it is not trivial to anticipate the effect of a little distress acting on the whole group.

DebtRank. Empirical application. The values of the credit exposures are unknown. However, the values of investments of each node $j$ in the equity of $i, Z_{j i}$, are available. We then take them as a starting point to construct a proxy of the impact of node $i$. We normalise their values, $\tilde{W}_{i j}=\frac{Z_{j i}}{\sum_{i} Z_{j l}}$. We further rescale them with respect to the maximum by means of an impact scaling factor, denoted as $\alpha \leq 1, W_{i j}=\alpha \frac{\tilde{W}_{i j}}{\max _{l}\left\{\tilde{W}_{l j}\right\}}$. In this way, the maximum impact on $j$ across the nodes $l$ equals $\alpha$. For instance if $\alpha=$

$0.2, j$ can withstand the default of $1 / 0.2=5$ or more counterparties before defaulting itself: a quite conservative scenario. Notice that the value $\alpha=0.2$ is the average ratio $A_{i j} / E_{i}$ that was observed at the end of 2007 in the Brazilian interbank network, the only case for which this information is published or accessible ${ }^{11}$. Therefore, we also run the experiments with $\alpha=0.2$. One could of course investigate other values of $\alpha$.

Moreover, $\alpha$ could also be made heterogeneous. Finally, we want to account for the fact that the market capitalization of the largest borrowers on the stock market decreased significantly in the first half of the time span of the data. We do so by rescaling the impact of a node on another accordingly, with the constraint that it does not exceed one. The final expression used for the impact is therefore,

$W_{i j}=\min \left\{1, \alpha \frac{\tilde{W}_{i j}}{\max _{l}\left\{\tilde{W}_{l j}\right\}} \frac{E_{j}(1)}{E_{j}(t)}\right\}$.

Bow-tie. A bow-tie architecture is a directed graph consisting of the following parts. The core is a strongly connected component (SCC). The IN consists of all nodes that can reach the SCC directly or indirectly via one or more directed paths. The OUT consists of all nodes that can be reached from the SCC directly or indirectly via one or more directed path. The Tubes and Tendrils (TT) consist of all nodes that are not in the SCC and are either reachable from the IN or/and can reach the OUT.

1. Schweitzer, F. et al. Economic networks: the new challenges. Science 325, 422-5 (2009).

2. Hidalgo, C., Klinger, B., Barabási, A. \& Hausmann, R. The product space conditions the development of nations. Science 317, 482 (2007).

3. Vitali, S., Glattfelder, J. B. \& Battiston, S. The network of global corporate control. PLOS-ONE 6 (2011).

4. Haldane, A. G. \& May, R. M. Systemic risk in banking ecosystems. Nature 469, 351-355 (2011)

5. De Masi, G., Iori, G. \& Caldarelli, G. Fitness model for the italian interbank money market. Phys. Rev. E 74, 066112 (2006).

6. Battiston, S., Glattfelder, J. B., Garlaschelli, D., Lillo, F. \& Caldarelli, G. The structure of financial networks. Network Science 131-163 (2010).

7. Battiston, S., Gatti, D., Gallegati, M., Greenwald, B. \& Stiglitz, J. Liaisons dangereuses: Increasing connectivity, risk sharing, and systemic risk. J. of Econ. Dyn. \& Control 36, 1121-1141 (2012).

8. Liu, Y.-Y., Slotine, J.-J. \& Barabasi, A.-L. Controllability of complex networks. Nature 473, 167-173 (2011).

9. Boss, M., Elsinger, H., Summer, M. \& Thurner, S. Network topology of the interbank market. Quant. Fin. 4, 677-684 (2004).

10. Soramäki, K., Bech, M. L., Arnold, J., Glass, R. J. \& Beyeler, W. E. The topology of interbank payment flows. Phys. A 379, 317-333 (2007).

11. Cont, R., Moussa, A. \& Santos, E. B. Network structure and systemic risk in banking systems. SSRN W.P. series (2010).

12. Mistrulli, P. Assessing financial contagion in the interbank market: Maximum entropy versus observed interbank lending patterns. J. of Banking \& Fin. 35, 1114-1127 (2011).

13. Opportunities exist to strengthen policies and processes for managing emergency assistance. GAO Tech. Report (2011).

14. Aoyama, H. et al. Pareto's law for income of individuals and debt of bankrupt companies. Fractals 8, 293-300 (2000).

15. Caldarelli, G. Scale-Free Networks: complex webs in nature and technology (Oxford University Press, 2007)

16. Garlaschelli, D., Battiston, S., Castri, M., Servedio, V. \& Caldarelli, G. The scale-free topology of market investments. Phys A 350, 491-499 (2005).

17. Battiston, S., Gatti, D. D., Gallegati, M., Greenwald, B. C. N. \& Stiglitz, J. E. Credit default cascades: when does risk diversification increase stability? J. of Fin. Stability, 8, 3, 138-149 (2012)

18. Lorenz, J., Battiston, S. \& Schweitzer, F. Systemic risk in a unifying framework for cascading processes on networks. EPJB 71, 441-460 (2009).

19. Nicosia, V., Criado, R., Romance, M., Russo, G. \& Latora, V. Controlling centrality in complex networks. Sci. Rep. (2012).

20. Pastor-Satorras, R. \& Vespignani, A. Epidemic spreading in scale-free networks. PRL 86, 3200-3203 (2001).

\section{Acknowledgments}

The authors acknowledge the financial support from the Swiss National Science Foundation Grant CR12I1-127000) and the European Commission FET Open Project "FOC" 255987. GC acknowledges the support from the Italian PNR project CRISIS-Lab and from the U.S. Department of the Defense, Defense Threat Reduction Agency, grant HDTRA1-11-1-0048.

\section{Author contributions}

SB supervised the work and wrote the main text. MP wrote the Supplementary Information. $\mathrm{SB}$ and MP carried out the numerical analyses and prepared the figures. MP and RK gathered the data. RK and PT contributed to the analyses and to writing the SI. GC contributed to the supervision of the work. All authors contributed to the editing of the manuscript and the interpretation of the results.

\section{Additional information}

Supplementary information accompanies this paper at http://www.nature.com/ scientificreports

Competing financial interests: The authors declare no competing financial interests.

License: This work is licensed under a Creative Commons

Attribution-NonCommercial-ShareAlike 3.0 Unported License. To view a copy of this license, visit http://creativecommons.org/licenses/by-nc-sa/3.0/

How to cite this article: Battiston, S., Puliga, M., Kaushik, R., Tasca, P. \& Caldarelli, G. DebtRank: Too Central to Fail? Financial Networks, the FED and Systemic Risk. Sci. Rep. 2, 541; DOI:10.1038/srep00541 (2012) 